\title{
Preference Integration and Optimization of Multistage Weighted Voting System Based on Ordinal Preference
}

\author{
Yu-ke Chen, ${ }^{1}$ Yan Zou, ${ }^{1}$ and Zhe Chen ${ }^{2}$ \\ ${ }^{1}$ School of Economics and Management, Chongqing Normal University, Chongqing 401331, China \\ ${ }^{2}$ Business School, Sichuan University, Chengdu 610064, China
}

Correspondence should be addressed to Zhe Chen; chenzhe@scu.edu.cn

Received 21 March 2014; Accepted 13 May 2014; Published 28 May 2014

Academic Editor: Chong Li

Copyright (C) 2014 Yu-ke Chen et al. This is an open access article distributed under the Creative Commons Attribution License, which permits unrestricted use, distribution, and reproduction in any medium, provided the original work is properly cited.

\begin{abstract}
Multistage voting is a common voting form through which the winners are selected. By virtue of weighted multistage voting rules, in this paper, we establish a weighted voting model by analyzing the correlation between individual preference and group preference. The weights of voters in each voting stage are adjusted through preference deviation degrees between individual preferences and group preference, and the ranking among candidates in each stage is determined according to weighted Borda function value. Examples are given to verify our model, which shows that weighted information aggregation model can mine more useful information from different individual preferences of voters to quicken the aggregation of group preference.
\end{abstract}

\section{Introduction}

Many social choice activities usually adopt multistage voting form. Multistage voting is a dynamic group decision-making procedure, in which the final winner is selected through round-by-round elimination. In multistage voting system, the final voting result is generated via multiple interrelated exhaustive processes. A voter can take part in multiple times of voting; one or more candidates are eliminated till only the qualified candidate is left. Ranking for candidates is a representation form of voters' individual preferences, which means a voter expresses both his favorite candidate and the ranking of other candidates in accordance with his preference. During multistage voting system, voters need to express their preference ranking in each voting stage, so it is a major issue to fairly and effectively aggregate the individual preferences into group preference.

Weighted multistage voting is a voting system embodying a voter's power on final result, which can embody the influences of different voters on the final result, so it is necessary to measure the power of voters. Common power indices include Shapley-Shubik power index [1], Banzhaf power index [1], and information entropy power index [2]. Existing studies on weighted voting mainly focus on simple voting form ("one person one vote") [3-5], while studies on weights measurement in weighted voting system are relatively fewer. Freixas and Kaniovski [6] proposed a new power index based on the minimum sum representation (MSR) of a weighted voting game. Cheng [7] gave "structure entropy weight method" under ranking conditions, which also combines Delphi method with fuzzy analysis method. Based on the concept of "Importance" of preference relation, Monroy and Fernández [8] proposed an extended Banzhaf index for weighted multicriteria simple games. Meanwhile, the ordinal equivalence of the Johnston, Banzhaf, and Shapley power indices is discussed in [9]. All above studies mainly focus on single stage group decision-making process with fixed decision makers and candidates.

Therefore this paper mainly discusses how to determine the weight of each voter in accordance with the relation between individual preferences and group preference in multistage voting system and puts forward relevant voting model. The less the deviation degree between individual preference of a voter and group preference is, the larger the weight of a voter will be, and vice versa. In fact, a voting process is a value judgment process not fussed about right or wrong, so we view the result (group preference) supported by most of the voters as right standard in certain period. With the 
changes of individual preferences and consequent changes of group preference in every round, determining weights of voters becomes a dynamic changing process referring to initial weight.

To facilitate our discussion, the paper is organized as follows. In Section 2, some definitions are given to support the following analysis about weighted voting. In Section 3, a method for determining weights of voters is presented subsequently. In Section 4, the voting model in multistage voting and an example for illuminating our ideas are given. At last, we conclude this paper with a summary and outlook for further research in Section 5.

\section{Preliminaries}

Definition 1. A binary relation $R$ on a set $A$, which is a subset of the domain $A \times A$, is a partial order denoted by " $>$ " if and only if $R$ is reflexive, symmetric, and transitive and is a weak partial order denoted by " $\square$ " if and only if $R$ is reflexive, complete, and transitive.

Definition 2. In individual preference, for two candidates $c_{i}$ and $c_{j}, c_{i}>c_{j}$ means that $c_{i}$ is greater than $c_{j}, c_{i} \square c_{j}$ means that there is no difference between $c_{i}$ and $c_{j}$, and $c_{i} \geq c_{j}$ means that $c_{i}$ is equal to and greater than $c_{j}$.

Definition 3. The order relation $c_{1}^{*}>c_{2}^{*}>\cdots>c_{n}^{*}$ means the partial order among the candidates set $\left\{c_{1}, c_{2}, \ldots, c_{n}\right\}$, where $c_{i}^{*}(i=1,2, \ldots, n)$ is the $i$ th $(i=1,2, \ldots, n)$ candidate.

Definition 4. Let $N\left(c_{i}>c_{j}\right)$ be the number of $c_{i}>c_{j}$ among all voters, $N\left(c_{i} \square c_{j}\right)$ the number of $c_{i} \square c_{j}$ among all voters, and $N\left(c_{i} \succeq c_{j}\right)$ the number of $c_{i} \succeq c_{j}$ among all voters.

There are many social choice functions, such as Condorcet function, Borda function, and Kemeney function to aggregate individual preferences into group preference. Different functions can generate different results. Borda function is "the best tool for acquiring all acceptance when an effective approach is sought for achieving a common objective and the fairest method when divergent opinions must be unified" [10].

Definition 5 (Borda number). If there are $m$ candidates, then Borda numbers for the candidates at No.1, No.2, .., No.m in the preference ranking will be $m-1, m-2, \ldots, 0$, respectively.

In essence, Borda number is the times of each candidate's superiority to other candidates in the preference rankings given by voters. In this paper, Borda numbers are used to express the utility value of each candidate in a given individual preference ranking.

\section{The Method of Determining Weights of Voters}

Let $V=\left\{v_{1}, v_{2}, \ldots, v_{m}\right\}$ be the set of voters, $C=\left\{c_{1}, c_{2}, \ldots, c_{n}\right\}$ the set of candidate, and $T$ the total stages in multistage voting. Let $M_{t}$ be the preference matrix of the voting stage $t(1 \leq t \leq T)$ which is constituted by all Borda numbers given by voters and $w^{t}=\left\{w_{1}^{t}, w_{2}^{t}, \ldots, w_{m}^{t}\right\}$ the weights vector of voters in Round $t ; m$ is the number of candidates participating in Round $t$.

Example 6. Let a voter's individual preference ranking for candidate set $C=\left\{c_{1}, c_{2}, c_{3}\right\}$ be $c_{1}>c_{3}>c_{2}$. Obviously, when $c_{1}$ is compared with $c_{2}$ and $c_{3}$, we have $c_{1}>c_{3}, c_{1}>c_{2}$, so $c_{1}$ is superior for 2 times and gets the utility value of 2 . When $c_{2}$ is compared with $c_{1}$ and $c_{3}$, we have $c_{1}>c_{2}, c_{3}>c_{2}$, so $c_{2}$ is superior for 0 times and gets the utility value of 0 . Similarly, $c_{3}$ gets the utility value of 1 .

Definition 7. Let $P_{t}$ be preference matrix of voters in the voting stage $t(t=1,2, \ldots, T) . P_{t}$ is denoted as

$$
P_{t}=\left(a_{i j}^{t}\right)_{m \times n}=\left[\begin{array}{cccc}
a_{11}^{t} & a_{12}^{t} & \ldots & a_{1 n}^{t} \\
a_{21}^{t} & a_{22}^{t} & \ldots & a_{2 n}^{t} \\
\ldots & \ldots & \ldots & \ldots \\
a_{m 1}^{t} & a_{m 2}^{t} & \ldots & a_{m n}^{t}
\end{array}\right],
$$

where $a_{i j}^{t}$ represents the utility value of the candidate $c_{i} \in C$ in individual preference ranking of the voter $v_{j} \in V$.

Example 8. Given the voter set $V=\left\{v_{1}, v_{2}, v_{3}, v_{4}\right\}$ and the candidate set $C=\left\{c_{1}, c_{2}, c_{3}\right\}$, in a specific voting stage $t$, preference rankings of all voters for the candidate set are $c_{1}>c_{2}>c_{3}, c_{1}>c_{3}>c_{2}, c_{2}>c_{1}>c_{3}$, and $c_{1}>c_{3}>c_{2}$. So, according to (8), we have

$$
P_{t}=\left[\begin{array}{llll}
2 & 2 & 1 & 2 \\
1 & 0 & 2 & 0 \\
0 & 1 & 0 & 1
\end{array}\right] .
$$

Definition 9. Borda function is defined as

$$
f_{B}\left(c_{i}\right)=\sum_{c_{j} \in C \backslash\left\{c_{i}\right\}} N\left(c_{i}>c_{j}\right),
$$

where $f_{B}\left(c_{i}\right)$ is the total times of superiority of candidate $c_{i}$ to other candidates and $C \backslash\left\{c_{i}\right\}$ expresses the candidate set exclusive of candidate $c_{i}$. So, according to Definition 7 , it can be known that the Borda function value of the candidate $c_{i}$ is the sum of row $i$ in preference matrix of voters.

Example 10. According to voters' preference given in Example 8 , Borda function values of candidates are as follows. Consider

$$
\begin{aligned}
& f_{B}\left(c_{1}\right)=N\left(c_{1}>c_{2}\right)+N\left(c_{1}>c_{3}\right)=7, \\
& f_{B}\left(c_{2}\right)=N\left(c_{2}>c_{1}\right)+N\left(c_{2}>c_{3}\right)=3, \\
& f_{B}\left(c_{3}\right)=N\left(c_{3}>c_{1}\right)+N\left(c_{3}>c_{1}\right)=2 .
\end{aligned}
$$

Definition 11. Deviation degree of individual preference, which means the departure of the individual preference from group preference, is defined as

$$
\operatorname{bias}_{j}^{t}=\frac{1}{m} \sqrt{\sum_{i=1}^{m}\left(a_{i j}^{t}-g_{i}^{t}\right)^{2}},
$$


where bias ${ }_{j}^{t}$ means the deviation degree of voter $v_{j}$ in voting stage $t, m(m \neq 0)$ is the number of candidates in stage $t$, and $g_{i}^{t}$ means the utility value of the candidate $c_{i}$ in group preference. Apparently, we have bias ${ }_{j}^{t} \geq 0$. The more bias ${ }_{j}^{t}$ approaches 0 , the smaller deviation degree of individual preference will be.

Definition 12. The amendatory value of weight for the voter $v_{j}$ in voting stage $t$ is denoted as

$$
\Delta w_{j}^{t}= \begin{cases}\frac{\left(\text { bias }_{\text {max }}^{t}-\text { bias }_{j}^{t}\right)}{\sum_{j=1}^{n}\left(\text { bias }_{\text {max }}^{t}-\text { bias }_{j}^{t}\right)}, & \sum_{j=1}^{n}\left(\text { bias }_{\text {max }}^{t}-\text { bias }_{j}^{t}\right) \neq 0 \\ 0, & \sum_{j=1}^{n}\left(\text { bias }_{\text {max }}^{t}-\text { bias }_{j}^{t}\right)=0,\end{cases}
$$

where bias ${ }_{\max }^{t}$ is the maximum among all amendatory values and $n$ is the number of voters. Obviously, we have $\Delta w_{j}^{t} \geq 0$.

Definition 13. The new weight of the voter $v_{j}$ after voting stage $t$ is denoted as

$$
w_{j}^{t+1}=\alpha * w_{j}^{t}+(1-\alpha) * \Delta w_{j}^{t},
$$

where $\alpha$ is weight correction coefficient for defining the amplitude of voter weight correction. $\alpha$ is a constant, and $\alpha \epsilon$ $[0,1]$. If $\alpha=1$, weights of voters will not be adjusted and will remain initial weights, while if $\alpha=0$, initial weights of voters will be neglected and will be determined only according to individual preference deviation in this voting stage.

After weights of voters are adjusted, voters closer to group preference can get larger weights, which will adequately indicate voter's individual value and facilitate concentration of group opinions.

\section{The Voting Model and an Example}

4.1. Analysis on Voting Process. A multistage voting process includes voting and votes counting. In this subsection, we express individual preferences by ranking and aggregate individual preferences into group preference by weighted Borda function.

Definition 14. Weighted Borda function is defined as

$$
f_{B^{\prime}}\left(c_{i}\right)=\sum_{c_{j} \in C \backslash\left\{c_{i}\right\}} \sum_{k=1}^{n}\left(N_{k}\left(c_{i}>c_{j}\right) * w_{k}\right),
$$

where $w_{k}$ is the weight of the voter $v_{k}$ and $N_{k}\left(c_{i}>c_{j}\right)$ means the number of $c_{i}>c_{j}$ for the voter $v_{k}$; obviously for the voter $v_{k}$, if $c_{i}>c_{j}$, we have $N_{k}\left(c_{i}>c_{j}\right)=1$ or else $N_{k}\left(c_{i}>c_{j}\right)=0$. Let $f_{B^{\prime}}\left(c_{i}\right)$ be the weighted Borda function in voting stage $t$. So, according to voter preference matrix and Definition 14 , we define $P_{t} w^{t}$ to express the vector of weighted Borda function of all candidates after voting stage $t$, where $P_{t}$ and $w^{t}$ express voter preference matrix of voting stage $t$ and postadjustment weights vector of voters, respectively.
Example 15. Suppose weights vector of the voter set $\left\{v_{1}, v_{2}\right.$, $\left.v_{3}, v_{4}\right\}$ is $w=(0.3,0.25,0.25,0.2)^{T}$; then the weighted Borda function values of candidates in Example 6 will be obtained according to (8). Consider

$$
\begin{aligned}
f_{B^{\prime}}\left(c_{1}\right) & =\sum_{k=1}^{n}\left(N_{k}\left(c_{1}>c_{2}\right) * w_{k}\right)+\sum_{k=1}^{n}\left(N_{k}\left(c_{1}>c_{3}\right) * w_{k}\right) \\
& =0.75+1=1.75, \\
f_{B^{\prime}}\left(c_{2}\right) & =\sum_{k=1}^{n}\left(N_{k}\left(c_{2}>c_{1}\right) * w_{k}\right)+\sum_{k=1}^{n}\left(N_{k}\left(c_{2}>c_{3}\right) * w_{k}\right) \\
& =0.25+0.55=0.8, \\
f_{B^{\prime}}\left(c_{3}\right) & =\sum_{k=1}^{n}\left(N_{k}\left(c_{3}>c_{1}\right) * w_{k}\right)+\sum_{k=1}^{n}\left(N_{k}\left(c_{3}>c_{1}\right) * w_{k}\right) \\
& =0+0.45=0.45 .
\end{aligned}
$$

In weighted multistage voting, each voter has an initial weight at first, but the voters more quickly getting close to group opinions will get more power with the increase of voting times; that is, voter weight will increase. On the contrary, the weight of voters deviating more from group opinions will decrease.

4.2. A Voting Algorithm. Basic principle of weighted multistage voting method is described as follows. At every voting stage, all voters give respective preference ranking for candidates firstly; then weighted Borda function values of candidates will be calculated based on adjusted weights and individual preference ranking of voters. If any candidate meets specified condition for win, the voting will end; otherwise the candidate with the lowest weighted Borda function value in this stage will be eliminated. Meanwhile, weights of voters will be readjusted according to the relation between individual preference and group preference at this stage, and then the next voting stage starts.

Algorithm 16. The steps are as follows.

Step 1. Let the voter set be $V=\left\{v_{1}, v_{2}, \ldots, v_{m}\right\}$, initial candidate set $C=\left\{c_{1}, c_{2}, \ldots, c_{n}\right\}, t=1$, and initial weights vector of voters $w^{t}=\left(w_{1}^{t}, w_{2}^{t}, \ldots, w_{n}^{t}\right)^{T}$.

Step 2. Obtain the voter preference matrix $P_{t}$ according to individual preference of voters at voting stage $t$.

Step 3. Calculate the weighted Borda function value $f_{B^{\prime}}^{t}\left(c_{i}\right)$ of candidate $c_{i}$ in voting stage $t$ according to (8) and corrected weights vector of voters $w^{t}$.

Step 4. If any candidate meets specified winning condition, the voting will end.

Step 5. Adjust voter's weights. According to (5), (6), and (7), we obtain new adjusted weights vector of voters $w^{t+1}=$ $\left(w_{1}^{t+1}, w_{2}^{t+1}, \ldots, w_{n}^{t+1}\right)$, which will be the weights vector of next voting stage. 
TABLE 1: Weights correction results of the first stage voting.

\begin{tabular}{lccccccccccc}
\hline & & & & & & & & & & \\
& $v_{1}$ & $v_{2}$ & $v_{3}$ & $v_{4}$ & $v_{5}$ & $v_{6}$ & $v_{7}$ & $v_{8}$ & $v_{10}$ \\
\hline bias $_{j}^{1}$ & 0.000 & 0.000 & 0.559 & 0.433 & 1.601 & 0.000 & 0.000 & 0.612 & 0.250 & 0.000 & 0.559 \\
$\Delta w_{j}^{1}$ & 0.129 & 0.129 & 0.061 & 0.077 & 0.000 & 0.129 & 0.129 & 0.055 & 0.099 & 0.129 & 0.061 \\
$w_{j}^{2}$ & 0.102 & 0.102 & 0.082 & 0.087 & 0.064 & 0.102 & 0.102 & 0.080 & 0.093 & 0.102 & 0.082 \\
\hline
\end{tabular}

Step 6. Eliminate the unique candidate with minimal weighted Borda function value. If there are several candidates with the same minimal weighted Borda function values, an additional voting will be held to select only one to eliminate.

Step 7. $t=t+1$, and turn to Step 2 for next voting stage.

4.3. An Example. Let the voter set be $V=\left\{v_{i} \mid i=1,2, \ldots\right.$, $11\}$, the candidate set $C=\left\{c_{1}, c_{2}, c_{3}, c_{4}\right\}$, and initial voter weight vector $w_{i}^{1}=1 / 11, i=1,2, \ldots, 11$. Winning condition in multistage voting is that the last one left in candidate set will be the winner through round-by-round elimination.

(1) Voting Stage 1. The preference rankings of voters are as follows. Consider

$$
\begin{array}{ll}
v_{1}: c_{1}>c_{4}>c_{2}>c_{3}, & v_{2}: c_{1}>c_{4}>c_{2}>c_{3}, \\
v_{3}: c_{2}>c_{1}>c_{4}>c_{3} & v_{4}: c_{4}>c_{1}>c_{3}>c_{2}, \\
v_{5}: c_{3}>c_{2}>c_{1}>c_{4}, & v_{6}: c_{1}>c_{4}>c_{2}>c_{3} \\
v_{7}: c_{1}>c_{4}>c_{2}>c_{3}, & v_{8}: c_{2}>c_{1}>c_{3}>c_{4}, \\
v_{9}: c_{4}>c_{1}>c_{2}>c_{3} & v_{10}: c_{1}>c_{4}>c_{2}>c_{3}, \\
v_{11}: c_{4}>c_{2}>c_{1}>c_{3} . &
\end{array}
$$

Hence we get the voter preference matrix:

$$
P_{1}=\left[\begin{array}{lllllllllll}
3 & 3 & 2 & 2 & 1 & 3 & 3 & 2 & 2 & 3 & 1 \\
1 & 1 & 3 & 0 & 2 & 1 & 1 & 3 & 1 & 1 & 2 \\
0 & 0 & 0 & 1 & 3 & 0 & 0 & 1 & 0 & 0 & 0 \\
2 & 2 & 1 & 3 & 0 & 2 & 2 & 0 & 3 & 2 & 3
\end{array}\right]
$$

According to (8), the weighted Borda function values of candidates are listed as follows:

$$
\begin{array}{ll}
f_{B^{\prime}}^{1}\left(c_{1}\right)=2.273, & f_{B^{\prime}}^{1}\left(c_{2}\right)=1.454 \\
f_{B^{\prime}}^{1}\left(c_{3}\right)=0.454, & f_{B^{\prime}}^{1}\left(c_{4}\right)=1.818
\end{array}
$$

Apparently, $f_{B^{\prime}}^{1}\left(c_{1}\right)>f_{B^{\prime}}^{1}\left(c_{4}\right)>f_{B^{\prime}}^{1}\left(c_{2}\right)>f_{B^{\prime}}^{1}\left(c_{3}\right)$, so the group preference is $c_{1}>c_{4}>c_{2}>c_{3}$. Therefore, $c_{3}$ is eliminated after the first round of voting, and the candidate set changes to $\left\{c_{1}, c_{2}, c_{4}\right\}$.

Next voter weights will be adjusted. Suppose weight correction coefficient and then individual preference deviation, weight correction, and postcorrection voter weight in voting stage 1 are calculated according to (5), (6), and (7), as shown in Table 1.

From Table 1 and $P_{1}$, it can be seen that individual preferences of candidates $v_{1}, v_{2}, v_{6}, v_{7}, v_{10}$ are consistent with group preference, so their individual preference deviation is 0 , and their weight corrections are the largest, and their weights all rise from 0.909 to 0.102 . While the voter $v_{5}$ has the largest individual preference deviation, so his weight correction is the smallest and becomes negative during weight adjustment. For example, weight of voter $v_{5}$ falls from 0.909 to 0.064 .

(2) Voting Stage 2. According to the performance of candidates in the second round voting, the voters give their preference rankings as follows:

$$
\begin{array}{ll}
v_{1}: c_{4}>c_{2}>c_{1}, & v_{2}: c_{4}>c_{2}>c_{1}, \\
v_{3}: c_{2}>c_{4}>c_{1}, & v_{4}: c_{1}>c_{4}>c_{2} \\
v_{5}: c_{2}>c_{4}>c_{1}, & v_{6}: c_{4}>c_{1}>c_{2}, \\
v_{7}: c_{4}>c_{1}>c_{2}, & v_{8}: c_{2}>c_{4}>c_{1} \\
v_{9}: c_{1}>c_{4}>c_{2}, & v_{10}: c_{4}>c_{1}>c_{2}, \\
v_{11}: c_{1}>c_{4}>c_{2} . &
\end{array}
$$

Hence the voter preference matrix is

$$
P_{2}=\left[\begin{array}{lllllllllll}
0 & 0 & 0 & 2 & 0 & 1 & 1 & 0 & 2 & 1 & 2 \\
1 & 1 & 2 & 0 & 2 & 0 & 0 & 2 & 0 & 0 & 0 \\
2 & 2 & 1 & 1 & 1 & 2 & 2 & 1 & 1 & 2 & 1
\end{array}\right]
$$

Next weighted Borda function values of candidates are calculated as follows:

$$
f_{B^{\prime}}^{2}\left(c_{1}\right)=0.831, \quad f_{B^{\prime}}^{2}\left(c_{2}\right)=0.657, \quad f_{B^{\prime}}^{2}\left(c_{4}\right)=1.512 .
$$

Obviously, $f_{B^{\prime}}^{2}\left(c_{4}\right)>f_{B^{\prime}}^{2}\left(c_{1}\right)>f_{B^{\prime}}^{2}\left(c_{2}\right)$, so the group preference is $c_{4}>c_{1}>c_{2}$. Therefore $c_{2}$ is eliminated after the second round voting, and the candidate set changes to $\left\{c_{1}, c_{4}\right\}$. Next weights of voters will be adjusted again. Suppose weight correction coefficient $\alpha=0.7$; then individual preference deviation, weight correction, and postcorrection voter weight in voting stage 2 are calculated, as shown in Table 2.

From Table 2 and $P_{2}$, it can be seen that individual preferences of $v_{6}, v_{7}, v_{10}$ are still fully consistent with group preference, so relevant voters also get the largest weight correction, and the weights of them all rise from 0.102 to 0.130 . While individual preferences of $v_{1}$ and $v_{2}$ are not fully consistent with group preference, so the weight of each of 
TABLE 2: Weights correction results of the second stage voting.

\begin{tabular}{lccccccccccc}
\hline & & & & & & & & & & \\
& $v_{1}$ & $v_{2}$ & $v_{3}$ & $v_{4}$ & $v_{5}$ & $v_{6}$ & $v_{7}$ & $v_{8}$ & $v_{9}$ & 0.000 \\
\hline bias $_{j}^{2}$ & 0.471 & 0.471 & 0.816 & 0.471 & 0.816 & 0.000 & 0.000 & 0.816 & 0.471 \\
$\Delta w_{j}^{2}$ & 0.083 & 0.083 & 0.000 & 0.083 & 0.000 & 0.196 & 0.196 & 0.000 & 0.083 & 0.196 & 0.083 \\
$w_{j}^{2}$ & 0.097 & 0.097 & 0.057 & 0.085 & 0.045 & 0.130 & 0.130 & 0.056 & 0.090 & 0.130 & 0.082 \\
\hline
\end{tabular}

TABLE 3: The relation of the value of $\alpha$ and voting results.

\begin{tabular}{lcccccccc}
\hline$\alpha$ & 0.99 & 0.97 & 0.95 & 0.9 & 0.89 & 0.85 & 0.7 & 0.5 \\
$f_{B^{\prime}}^{2}\left(c_{1}\right)$ & 0.459 & 0.465 & 0.476 & 0.497 & 0.501 & 0.517 & 0.573 & 0.639 \\
$f_{B^{\prime}}^{2}\left(c_{4}\right)$ & 0.541 & 0.535 & 0.503 & 0.503 & 0.499 & 0.482 & 0.427 & 0.361 \\
\hline
\end{tabular}

them falls from 0.102 to 0.097 . The weights variation of other voters is in a similar way.

(3) Voting Stage 3. According to the performance of candidates in voting stage 3 , the voters give their preference rankings as follows:

$$
\begin{array}{ll}
v_{1}: c_{1}>c_{4} 1, & v_{2}: c_{4}>c_{1}, \\
v_{3}: c_{4}>c_{1}, & v_{4}: c_{1}>c_{4} \\
v_{5}: c_{4}>c_{1}, & v_{6}: c_{1}>c_{4}, \\
v_{7}: c_{1}>c_{4}, & v_{8}: c_{4}>c_{1} \\
v_{9}: c_{4}>c_{1}, & v_{10}: c_{1}>c_{4}, \\
v_{11}: c_{4}>c_{1} . &
\end{array}
$$

So we have

$$
\begin{aligned}
& P_{3}=\left[\begin{array}{lllllllllll}
1 & 0 & 0 & 1 & 0 & 1 & 1 & 0 & 0 & 1 & 0 \\
0 & 1 & 1 & 0 & 1 & 0 & 0 & 1 & 1 & 0 & 1
\end{array}\right], \\
& f_{B^{\prime}}^{2}\left(c_{1}\right)=0.573, \\
&
\end{aligned}
$$

Because $f_{B^{\prime}}^{3}\left(c_{1}\right)>f_{B^{\prime}}^{3}\left(c_{4}\right)$, we have $c_{1}>c_{4}$. Therefore the final winner is $c_{1}$, in voting stage 3 , although only 5 voters among 11 voters prefer $c_{1}$, while the remaining 6 voters prefer $c_{4}$. In consideration of voter weights, all voters with heavy weight inclusive of $v_{1}, v_{7}, v_{10}$ elect $c_{1}$ in the last round of voting, so candidate $c_{1}$ becomes the final winner.

Note. During the dynamic adjustment of voter weights, the amplitude of weights adjustment is related to weight correction coefficient $\alpha$. Table 3 shows the relation between variation of $\alpha$ value and the result of the last round of voting in the above example. From Table 3, it can be seen that when $\alpha \geq 0.9$ and $1-\alpha<0.1$, initial weights play a main role, while the influence of weight adjustment on weight variation is too small, so weighted voting result is almost the same as voting without considering weights. But voter weights will influence voting result when $\alpha<0.9$ and $1-\alpha \geq 0.1$. Normally, rather small weight adjustment cannot embody the influence of voter weight on voting result, but overlarge adjustment may exaggerate the role of voter weights, so $\alpha$ value should be determined according to historical experience and expert judgment.

The following conclusions can be drawn from above analysis. (1) In weighted voting mode, voting result is not only related to preference rankings of candidates but also influenced by variation of voter weight. (2) Adjustment of voter weight depends on weight correction coefficient $\alpha$ which can be determined according to historical experience and expert judgment. (3) Weighted multistage voting can quicken the aggregation of preference information and improve decisionmaking efficiency.

\section{Conclusion}

The paper focuses on multistage voting model based on ordinal individual preferences through dynamically adjusting weights of voters. Because performance of candidates and cognition of voters differ, preference rankings of voters may be inconsistent. Weighted information aggregation mode can mine more useful information from different individual preference rankings of voters and quicken the aggregation of group preference. Weights of voters at each voting stage are determined mainly through the proximity between individual preferences and group preference, so weights of voters in every voting stage are adjusted, and then weighted Borda function values are calculated to decide the ranking of this stage. We know that (1) when weights of voters are considered, the candidate eliminated in every round and the final winner depend on not only traditional preference aggregation functions, such as Borda function and Cordorcet function, but also voters' weights of every round; (2) weights of voters in every stage are related to both its initial value and the proximity between individual preference and group preference. Weighted multistage voting model can quicken information aggregation and cut decision-making cost. There are some other related questions needed to be studied in our future work. Ordinal preferences are our research bases in this paper, while weighted voting models based on other preference forms are also worthy to study. Meanwhile the weighted voting under strategy condition is our future work. 


\section{Conflict of Interests}

The authors declare that there is no conflict of interests regarding the publication of this paper.

\section{Acknowledgments}

This work is partially supported by the National Science Foundation of China (no. 11001289), Ministry of Education in China Project of Humanities and Social Sciences (13YJC630252), Chongqing Natural Science Foundation, China (CSTC2010BB9321), and the fund of Chongqing Normal University, China (11XWB016).

\section{References}

[1] L. S. Shapley and M. Shubik, "A method for evaluating the distribution of power in a committee system," The American Political Science Review, vol. 48, no. 3, pp. 787-792, 1954.

[2] E. T. Jaynes, "Information theory and statistical mechanics. II," Physical Review, vol. 108, no. 2, pp. 171-190, 1957.

[3] M. Sutter, "Dan S. Felsenthal and Moshé Machover, the measurement of voting power: theory and practice, problems and paradoxes," Public Choice, vol. 102, no. 3, pp. 373-376, 2000.

[4] P. D. Straffin, "Homogeneity, independence, and power indices," Public Choice, vol. 30, no. 1, pp. 107-118, 1977.

[5] A. Taylor and A. Pacelli, Mathematics and Politics: Strategy, Voting, Power, and Proof, Springer, New York, NY, USA, 2008.

[6] J. Freixas and S. Kaniovski, "The minimum sum representation as an index of voting power," European Journal of Operational Research, vol. 233, no. 3, pp. 739-748, 2014.

[7] Q. Y. Cheng, "Structure entropy weight method to confirm the weight of evaluating index," System Engineering Theory and Practice, vol. 30, no. 7, pp. 1225-1228, 2010.

[8] L. Monroy and F. R. Fernández, "Banzhaf index for multiple voting systems. An application to the European Union," Annals of Operations Research, vol. 215, no. 1, pp. 215-230, 2014.

[9] J. Freixas, D. Marciniak, and M. Pons, "On the ordinal equivalence of the Johnston, Banzhaf and Shapley power indices," European Journal of Operational Research, vol. 216, no. 2, pp. 367-375, 2012.

[10] M. Dummett, "The Borda count and agenda manipulation," Social Choice and Welfare, vol. 15, no. 2, pp. 289-296, 1998. 


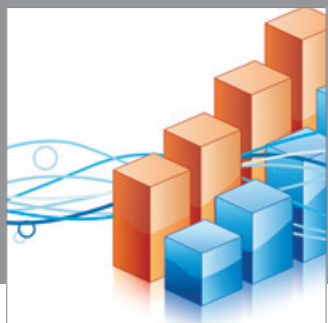

Advances in

Operations Research

mansans

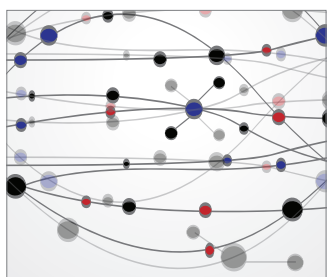

The Scientific World Journal
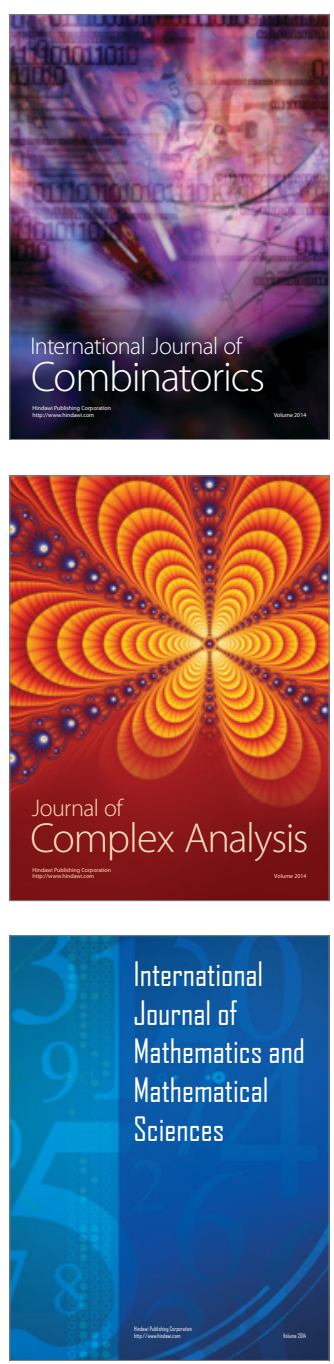
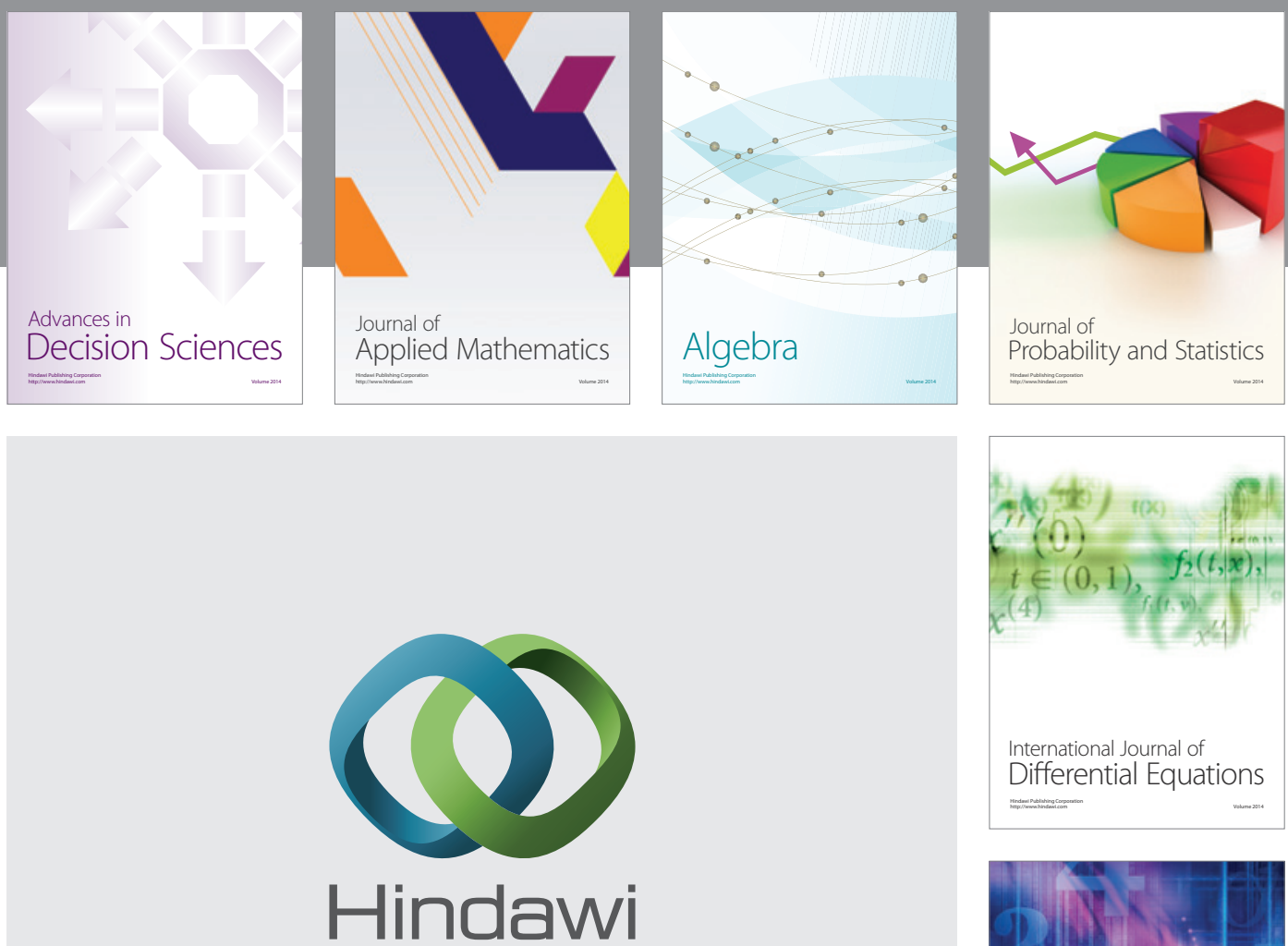

Submit your manuscripts at http://www.hindawi.com
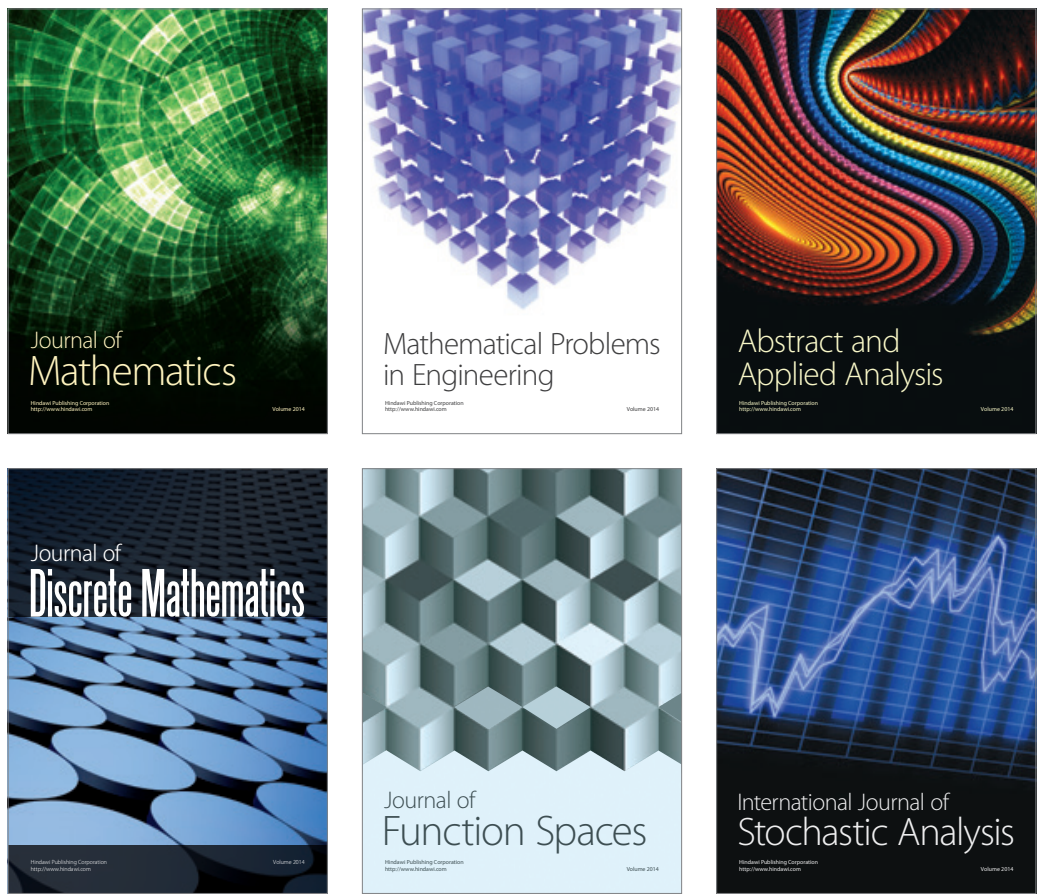

Journal of

Function Spaces

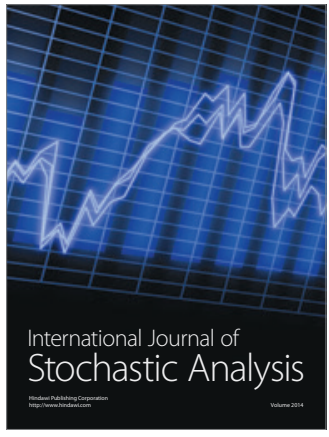

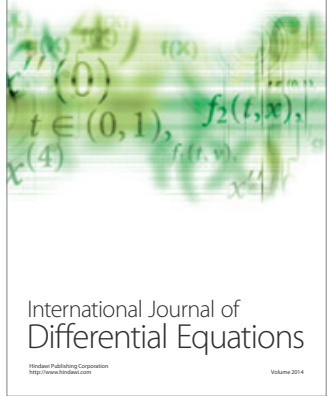
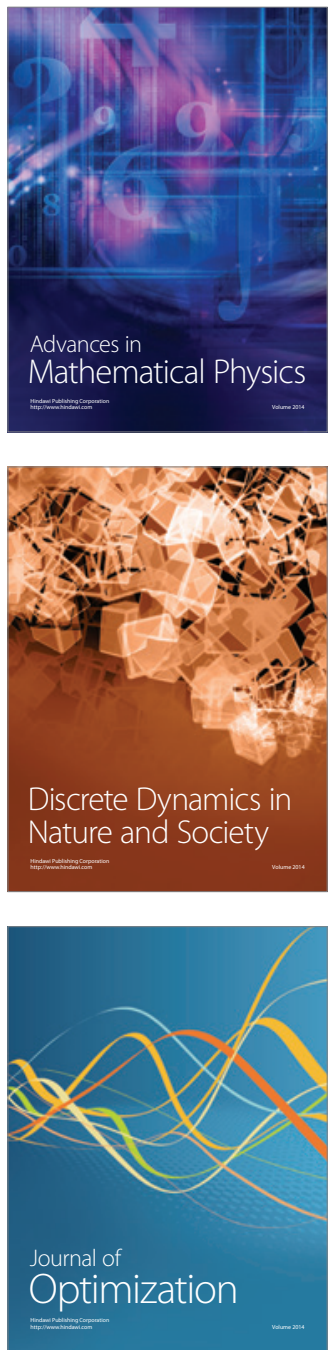